$\begin{array}{cl}\begin{array}{c}\text { Revue } \\ \text { de Ihistoire }\end{array} & \text { Revue de l'histoire des religions } \\ \text { des religions } & 2 \mid 2010 \\ & \text { Varia }\end{array}$

\title{
Une gigantomachie dans la Genèse ? Géants et héros dans les textes bibliques compilés
}

Is there a gigantomachy in Genesis? Giants and heroes in the compiled biblical texts

\section{Christophe Lemardelé}

\section{OpenEdition}

\section{Journals}

Édition électronique

URL : http://journals.openedition.org/rhr/7572

DOI : $10.4000 /$ rhr.7572

ISSN : 2105-2573

Éditeur

Armand Colin

Édition imprimée

Date de publication : 1 juin 2010

Pagination : 155-174

ISBN : 978-2200-92656-4

ISSN : 0035-1423

\section{Référence électronique}

Christophe Lemardelé, « Une gigantomachie dans la Genèse ? Géants et héros dans les textes

bibliques compilés », Revue de l'histoire des religions [En ligne], 2 | 2010, mis en ligne le 01 juin 2013, consulté le 19 avril 2019. URL : http://journals.openedition.org/rhr/7572 ; DOI : 10.4000/rhr.7572 


\section{Une gigantomachie dans la Genèse? Géants et héros dans les textes bibliques compilés}

Les " géants 》 de la Genèse précédant le Déluge ne peuvent être assimilés au thème de la gigantomachie grecque. Ils se rattachent en effet à une conception ouest-sémitique attribuant une taille impressionnante à des héros légendaires qui ne s'opposaient nullement au monde divin. Ces héros, qui devenaient les dieux du Shéol à leur mort, sont nombreux et divers dans les textes bibliques, ce qui permet d'entrevoir, d'une part, qu'il y avait une profusion de récits hérö̈ques dans la culture israélitojudahite, d'autre part, que la "fonction" guerrière était un des fondements de cette culture ancienne. La résurgence de tels géants dans la littérature judéenne foisonnante à l'époque hellénistique et romaine rend compte de conceptions théologiques qui n'ont plus rien à voir avec cette fonction.

\section{Is there a gigantomachy in Genesis? \\ Giants and heroes in the compiled biblical texts}

The "giants" of the Genesis preceding the Flood cannot be assimilated to the Greek theme of gigantomachy. In fact, they are connected to a westsemitic conception giving an amazing size to legendary heroes who were not opposed at all to the divine world. These heroes who became the gods of the Sheol after their deaths, are many and various in the Biblical texts which allows us to think, on the one hand that there was an abundance of heroic stories in the "Israelito-Judahite" culture, on the other hand that the warrior "function" was one of the basis of this ancient culture. The resurgence of such giants in the Judean literature teeming during the Hellenistic and Roman times renders an account of theological conceptions which have nothing to do with this function anymore. 
"Alors que les hommes avaient commencé à se multiplier sur la surface du sol et que des filles leur étaient nées, les fils de Dieu virent que les filles d'homme étaient belles et ils prirent pour femmes celles de leur choix. Le Seigneur dit : "Mon esprit ne dirigera pas toujours l'homme, étant donné ses erreurs : il n'est que chair et ses jours seront de cent vingt ans." En ces jours, les géants étaient sur la terre et ils y étaient encore lorsque les fils de Dieu vinrent trouver des filles d'homme et eurent d'elles des enfants. Ce sont les héros d'autrefois, ces hommes de renom » (Genèse 6, 1-4) ${ }^{1}$.

Dans le grand récit mythologique de la Genèse, il est un épisode qui suscite bien des interrogations tant il évoque une mythologie polythéiste et tant il surprend par son laconisme. Il s'agit bien entendu de l'accouplement entre les fils de Dieu et les filles des hommes (Genèse 6), union impropre semblant «accoucher» de géants et, ainsi, déclencher le déluge. Le « micro-récit» conduit à trois types de démarches : d'abord et en aval, à la comparaison avec la littérature judéenne tardive (pseudépigraphique) dans laquelle on retrouve l'épisode considérablement plus développé; ensuite et en amont, à la comparaison avec la littérature mésopotamienne dans laquelle se trouvent des récits de déluge bien plus anciens; enfin et aussi, à la comparaison avec la mythologie grecque qui fait état de géants dans les temps primordiaux.

Les questions centrales qui sont posées à partir de ces comparaisons sont de savoir s'il y avait une tradition hénochienne parallèle aux récits de la Genèse qui n'y ferait qu'allusion, de savoir si cette tradition a son origine ou non dans la mythologie mésopotamienne au sens large et de se poser la question des interactions entre les géants grecs et les géants bibliques. Ces questions n'obtiennent pas pour l'instant de réponses très satisfaisantes ou, lorsque les réponses sont tranchées, le consensus ne se fait pas autour d'elles. Par exemple, il est encore bien difficile de savoir avec certitude si 1Hénoch 6-11 développe Genèse 6, 1-4 ou bien si le second " texte » dépend du premier ou résume une source commune aux deux ${ }^{2}$. Or il nous semble qu'avant de s'interroger sur ces problèmes

1. Traduction œcuménique de la Bible, Paris, 1988, p. 60-61.

2. Après l'audacieuse suggestion de Jósef Tadeusz Milik, The Books of Enoch. Aramaic Fragments of Qumrân Cave 4, Oxford, 1976, p. 30-32, qui faisait du texte biblique l'emprunteur, la majorité des savants pense plutôt à un déve- 
si intéressants, il est nécessaire de bien analyser, textuellement et littérairement, le court «fragment» biblique pour, ensuite, chercher des géants présents dans d'autres textes bibliques en étudiant les mentions relevées en fonction de l'histoire rédactionnnelle des différents livres. Il s'agit donc de mettre en évidence la conception mythique des géants qu'ont pu avoir les différents auteurs israélites et judahites, cela dans une dimension diachronique, c'est-à-dire en tentant d'ordonner chronologiquement le dossier ${ }^{3}$. Quand nous aurons bien identifié cette conception et son évolution, peut-être alors sera-t-il plus aisé d'envisager les comparaisons de corpus à corpus, de tradition à tradition et de culture à culture.

\section{GENÈSE 6, 1-4 : NEPHILIM ET GIBboRIM}

Élaborée au cours du XIX ${ }^{e}$ siècle, la théorie documentaire permettait de rendre compte de l'aspect composite du Pentateuque, en identifiant quatre strates rédactionnelles ( $\mathrm{J}, \mathrm{E}, \mathrm{P}$ et $\mathrm{D})$, et proposait des

loppement hénochien à partir du texte biblique : voir notamment Mathias Delcor, « Le mythe de la chute des anges et de l'origine des géants comme explication du mal dans le monde dans l'apocalyptique juive. Histoire des traditions ", Revue de l'histoire des religions 190 (1976), p. 3-53; George W.E. Nickelsburg, 1 Enoch 1, (Hermeneia), Minneapolis, 2001, p. 166-169; Loren T. Stuckenbruck, « The « Angels » and « Giants » of Genesis 6:1-4 in Second and Third Century BCE Jewish Interpretation: Reflections on the Posture of Early Apocaliptic Traditions », Dead Sea Discoveries, 7, 2000, p. 354-377; James C. Vanderkam, « The Interpretation of Genesis in 1 Enoch », P.W. Flint (éd.), The Bible at Qumran. Text, Shape and Interpretation, Grand Rapids, 2001, p. 129-148; Devorah Dimant, «1 Enoch 6-11 : A Fragment of a Parabiblical Work », Journal of Jewish Studies, 53, 2002, p. 223-237; Helge S. Kvanvig, « The Watcher Story and Genesis. An Intertextual Reading », Scandinavian Journal of the Old Testament, 18, 2004, p. 163-183. Bien que beaucoup d'auteurs reconnaissent que les liens entre les deux textes ne se réduisent pas au pur et simple emprunt, peu d'entre eux estiment qu'il puisse y avoir deux traditions parallèles et opposées : voir pour cette thèse Andreas Bedenbender, «Traces of Enochic Judaism within the Hebrew Bible », Henoch, 24, 2002, p. 39-48.

3. Notre approche sera dans la filiation de celle de Rüdiger Bartelmus, Heroentum in Israel und seiner Umwelt, (Abhandlungen zur Theologie des Alten und Neuen Testament 65), Zürich, 1979, considérant plutôt le «fragment » de Genèse comme un mythe étiologique. Le dossier a été également traité par Lothar Perlitt, «Riesen im Alten Testament », Deuteronomium-Studien, (Forschungen zum Alten Testament 8), Tübingen, 1994, p. 205-246, mais dans une optique distincte puisque l'auteur ne reconnaît aucune conception héroïque dans des mentions qu'il juge trop tardives. 
datations ${ }^{4}$. Désormais, le consensus n'est plus accepté, le rédacteur élohiste (E) a disparu et le yahwiste (J), quand il est encore considéré comme un éditeur, n'est plus dénommé ainsi que par convention ${ }^{5}$. Les cinq premiers livres de la Bible ne sont plus tant isolés des autres pour former un corpus qui n'a dû être qu'artificiel et tardif, ils sont rattachés au livre de Josué (Hexateuque) ou aux autres livres « historiques » (Ennéateuque), et la Genèse est de plus en plus considérée comme le dernier ajout d'importance à l'édifice 6 . Il ne reste plus beaucoup de certitudes en ce qui concerne les datations si ce n'est que la constitution du livre n'a pu se faire que bien après la chute du royaume d'Israël (fin du VIII ${ }^{e}$ siècle) et même bien après celle de Juda (début $\mathrm{du} \mathrm{VI}^{\mathrm{e}}$ siècle). Les périodes perse et hellénistique (Lagide) sont le plus souvent retenues par les exégètes ce qui permet d'affirmer que le Pentateuque comme corpus n'a existé que peu de temps avant sa traduction en grec au $\mathrm{III}^{\mathrm{e}}$ siècle av.n.è. (Septante) ${ }^{7}$.

De la théorie documentaire, ne subsistent que les strates rédactionnelles D (Deutéronome et deutéronomiste) et P (Priesterschrift), essentiellement vues comme des compositions émanant d'écoles distinctes, l'une « laïque » $\left(\mathrm{K}^{\mathrm{D}}\right)$, l' autre sacerdotale $\left(\mathrm{K}^{\mathrm{P}}\right)$, leur combinaison étant le fruit d'un compromis entre elles afin de produire un document de référence pour le judaïsme naissant à l'époque perse ${ }^{8}$, et la seconde élaborant le grand « mythe historicisant » des origines ${ }^{9}$. Le modèle proposé est séduisant mais il ne permet toutefois pas de rendre complètement compte de la réalité si complexe et si variée des

4. Voir Thomas Römer, «La formation du Pentateuque: histoire de la recherche », T. Römer, J.-D. Macchi, C. Nihan (éd.), Introduction à l'Ancien Testament, (le Monde de la Bible 49), Genève, 2004, p. 67-84.

5. Christoph Levin, "The Yahwist : The Earliest Editor in the Pentateuch », Journal of Biblical Literature, 126, 2007, p. 209-230.

6. Voir les études réunies dans Thomas Römer, Konrad Schmid (éd.), Les Dernières Rédactions du Pentateuque, de l'Hexateuque et de l'Ennéateuque, (Bibliotheca Ephemeridum Theologicarum Lovaniensium 203), Leuven, 2007.

7. Pour cette traduction et ses raisons se détachant de la légende des soixantedouze traducteurs exposée dans la Lettre d'Aristée, voir dernièrement l'étude de Jan Joosten, "Le milieu producteur du Pentateuque grec », Revue des études juives, 165, 2006, p. 349-361.

8. T. Römer, "La construction du Pentateuque », T. Römer, K. Schmid, Les Dernières Rédactions du Pentateuque, p. 17-18, résumant la thèse de Erhard Blum, Studien zur Komposition des Pentateuch, (Beihefte zur Zeitschrift für die Alttestamentliche Wissenschaft 189), Berlin-New York, 1990.

9. Christophe Nihan, « L'écrit sacerdotal entre mythe et histoire », G.J. Brooke, T. Römer (éd.), Ancient and Modern Scriptural Historiography, (Bibliotheca Ephemeridum Theologicarum Lovaniensium 207), Leuven, 2007, p. 151-190. 
textes. De manière extrêmement simplifiée, on peut accepter l'idée que des auteurs sacerdotaux - au sens large du terme : des prêtres, ce qui comprend les sources $\mathrm{H}$ (Lévitique 17-26), $\mathrm{P}^{(\mathrm{s})}$ et autres aient écrit et constitué les quatre premiers livres bibliques pour finir par les rattacher au Deutéronome et former ainsi le Pentateuque. Dans cette optique, $P$ aurait édité des blocs existants que nous appelions J ou E auparavant. Cependant, pour le cas qui nous occupe, la recherche exégétique récente ne considère pas la péricope comme étant un fragment ancien encadré par des strates rédactionnelles sacerdotales. À l'instar de l'histoire de la tour de Babel (Genèse 11, 1-9), le récit est vu comme étant une interpolation insérée lors des dernières phases de rédaction, soit aux IV ${ }^{\mathrm{e}}-\mathrm{III}^{\mathrm{e}}$ siècles $^{10}$. Ces courtes narrations peuvent être d'ailleurs comprises à l'aune de l'influence grecque et de la littérature mésopotamienne ${ }^{11}$.

Le récit intervient après la longue liste généalogique qui va d'Adam à Noé en passant par Hénoch (Gn 5). Il est censé justifier le déluge qui n'aurait donc pas eu de cause précise dans le récit originel et dans sa relecture sacerdotale $(\mathrm{P})^{12}$. La mention des « fils d'Elohim » ne nous intéresse pas au premier chef car elle nous entraînerait vers la question du polythéisme suggéré dans ce passage. Nous préférons nous attarder sur le verset 4 et son peu de rapport avec le verset 2 qui fait état de l'union des fils d'Elohim et des filles d'Adam ${ }^{13}$. En effet, les géants ne sont en aucun cas le résultat de cette union lorsqu'on lit le texte attentivement, cela quelle que soit la version. L'hébreu du texte massorétique comme le grec de la Septante donnent l'impression d'un sommaire et le grec est encore plus confus :

10. Pour l'étude rédactionnelle de la première partie de la Genèse, voir Markus Witte, Die Biblische Urgeschichte: Redaktions- und theologiegeschichtliche Beobachtungen zu Genesis 1, 1-11, 26, (Beihefte zur Zeitschrift für die Alttestamentliche Wissenschaft 265), Berlin, 1998.

11. Albert de Pury, « $\mathrm{P}^{\mathrm{G}}$ as the Absolute Beginning », T. Römer, K. Schmid, Les Dernières Rédactions du Pentateuque, p. 114-115.

12. Ce qui expliquerait pourquoi la structure narrative du texte manque de cohérence : H.S. Kvanvig, "Gen 6, 1-4 as an Antediluvian Event », Scandinavian Journal of the Old Testament, 16, 2002, p. 79-112 (spécialement p. 81-86).

13. Distinction que ne fait pas Ronald S. Hendel, «Quand les fils de Dieu s'unissaient aux filles des hommes ", H. Shanks (dir.), L'Aventure des manuscrits de la mer Morte, Paris, 1996 (New York, 1992), p. 213-224. Il relève les allusions polythéistes (assemblée divine) contenues dans les textes bibliques mais sans remarquer qu'elles ne font jamais état de géants. Autrement dit, Gen 6, 1-4 est le seul texte biblique qui évoque monde divin et géants ensemble. 
«Or les géants étaient sur la terre en ces jours-là; et après cela, quand les fils de Dieu s'approchaient des filles des hommes et qu'ils engendraient pour eux-mêmes, c'étaient là les géants du temps passé, les hommes fameux ${ }^{14}$. »

Il y a la mention des jours anciens ( 'wlm/aiôn) à la fin du verset qui semblent antérieurs aux jours (bymym/en tais hêmerais) pendant lesquels l'épisode s'est déroulé. Les géants étaient donc sur la terre avant, pendant mais aussi après (wgm '凶ry-kn/kai met' ekeino) l'union entre les entités divines et humaines. Le verbe «être » de début de verset est à l'accompli en hébreu et à l'imparfait en grec, ce qui a pour effet de briser l'élan du récit. Il faut finir en disant que les fils d'Elohim ne s'accouplèrent avec les filles d'Adam que dans le but d'engendrer pour eux-mêmes (wyldw lhm hmh/egennôsan heautois ekeinoi). Aussi étrange que cela puisse paraître, il semble que la mention des géants ne soit que le rappel d'un « âge d'or », époque pendant laquelle il y eut cette union illicite et hybride. Le verset les concerne d'ailleurs, l'union n'étant rappelée que pour mentionner les fils d'Elohim et leur désir égoïste de filiation, et il forme en quelque sorte un chiasme :

« Les nephilîm étaient sur la terre ces jours-là, et aussi après que les fils d'Elohim étaient venus vers les filles de l'homme et n'avaient enfanté pour eux-mêmes, les gibbōrîm qui [étaient] d'autrefois, des hommes de renom ${ }^{15}$. »

L'autre aspect très intéressant de ce verset se trouve dans la mention de deux termes que le grec a rendus assez improprement par gigantes. Le traducteur n'avait sans doute pas le choix pour rendre le premier terme (nplym) par «géants» mais le second (gbrym), bien que synonyme du premier dans le verset, aurait mérité une nuance de sens. Rendre nephilîm par gigantes se comprend mieux encore par la mention en Nombres 13, 33 d'Anaqim appelés ainsi et décrits comme de taille bien supérieure aux hommes. En revanche, la traduction grecque de Genèse 10, 8-9, basée très probablement sur le verset 4 qui nous occupe, ne peut convenir pour Nemrod qui n'est pas décrit comme un géant mais qualifié de chasseur $(g b w r \otimes$ $y d$ ). Chercher à comprendre le sens précis de ces deux termes tout

14. Traduction de Marguerite Harl, La Genèse, (La Bible d'Alexandrie 1), Paris, 1986, p. 126.

15. Traduction littérale de l'hébreu. 
en les situant dans leur contexte culturel pourra nous aider à saisir quelle était la conception du passé mythique évoqué dans ce verset. Peut-être ne s'agit-il pas d'une conception empruntée comme c'est le cas pour le déluge, ni même d'un épisode plus ou moins conjoncturel comme l'union des filles d'homme et des fils de Dieu pour insister sur les raisons du cataclysme. Il est en effet possible qu'il faille penser à une conception mythique propre aux auteurs des différents textes bibliques.

\section{Nombres 13 et DeUteronome 2-3 : NePHILIM ET RePHa' 'M}

Le terme de nephilîm ne se retrouve que dans le quatrième livre du Pentateuque. Le livre des Nombres est si hétéroclite qu'il est de plus en plus considéré comme ayant été élaboré tardivement à partir de matériaux divers, cela afin de constituer le Pentateuque ${ }^{16}$, construction semble-t-il préférée à terme à une construction incluant les livres « historiques » (Hexateuque ou Ennéateuque) par les derniers rédacteurs ${ }^{17}$. On trouve le terme, qui plus est, dans un passage qui semble repris de Deutéronome 1, comme l'on trouve en Nombres des préoccupations et des motifs deutéronomiques ${ }^{18}$. Bien que des parallèles existent entre l'ensemble constitué Deutéronome 1-3 et l'ensemble disparate Nombres 13-14, 21 et 32, les spécialistes du livre n'en déduisent pas des cas d'intertextualité directe entre les deux, présupposant plutôt des traditions anciennes insérées, relues,

16. C. Nihan, T. Römer, «Le débat actuel sur le Pentateuque », T. Römer, J.-D. Macchi, C. Nihan, Introduction à l'Ancien Testament, p. 105, résumant l'étude de Reinhard Achenbach, Die Vollendung der Tora: Studien zur Redaktionsgeschichte des Numeribuches im Kontext von Hexateuch und Pentateuch, (Beihefte zur Zeitschrift für Altorientalische und Biblische Rechtsgeschichte 3) Wiesbaden, 2003.

17. Cependant, s'il s'avère que ce travail rédactionnel a trouvé un prolongement en Josué, comme l'avance C. Nihan, « La mort de Moïse (Nb 20, 1-13; 20, 22-29; 27, 12-23) et l'édition finale du livre des Nombres », T. Römer, K. Schmid, Les Dernières Rédactions du Pentateuque, p. 145-182, alors la notion de Pentateuque ou de Torah n'a peut-être pas réellement de sens avant la Septante.

18. À ce titre, la thèse d'Achenbach prolonge en quelque sorte celle de Blum en présupposant l'activité de rédacteurs post-sacerdotaux, ayant des visées « théocratiques », fondant les styles de $\mathrm{P}$ et de $\mathrm{D}$ dans le livre des Nombres. Voir T. Römer, « Nombres », T. Römer, J.-D. Macchi, C. Nihan, Introduction à l'Ancien Testament, p. 206. 
corrigées et prolongées ${ }^{19}$. Il est toutefois intéressant de noter que $\mathrm{Nb} 13$ et $\mathrm{Dt} 1,19$ ss font mention d'une exploration du pays de Canaan pour l'un et de la montagne des Amorites et des terres situées au nord de Qadesh-Barnéa pour l'autre (Dt 1, 7 et 19). De même, $\mathrm{Nb}$ 21, 10ss et $\mathrm{Dt}$ 2, 24-3, 7 relatent parallèlement mais de manière distincte la montée du peuple d'Israël au pays de Moab et ses guerres victorieuses contre Sihôn l'Amorite, roi de Heshbôn, et contre Og, roi du Bashân ${ }^{20}$. De là suit l'installation de certaines tribus dans les territoires transjordaniens nouvellement conquis, cela en $\mathrm{Nb} 32$ et en $D t 3,8-17$. Il y a donc bien une répétition des mêmes épisodes que l'on ressent dans le Deutéronome lorsque l'on procède par une lecture linéaire des livres mais qui est bien plus due au livre des Nombres ${ }^{21}$. En effet, les récits dans ce livre sont éloignés les uns des autres, ils sont bien souvent amplifiés par rapport à ceux de Deutéronome et il semble même qu'il y ait eu un ajout dans ce périple transjordanien avec la guerre contre les Madianites (Nombres 31$)^{22}$. En outre, il faut noter l'aspect incongru de textes comme ceux de $\mathrm{Nb}$ 13-14 qui font état d'une exploration du pays par le Sud, devançant de manière assez artificielle la véritable conquête qui se fera par l'Est. Cependant, les styles narratifs sont différents dans les deux livres et $N b 21$ se réfère à un « livre des guerres de Yhwh» (v. 14) et aux « poètes ${ }^{23} »($ v. 27) comme à des sources. Si l'on ajoute à cela la possibilité que le Deutéronome ait pu être d'origine sichémite et rattaché après la constitution d'un Tétrateuque, et donc après l'élaboration de Nombres, alors, en effet, toute filiation directe $\mathrm{Dt}-\mathrm{Nb}$ semble exclue ${ }^{24}$.

19. Voir déjà l'étude d'Olivier Artus, Études sur le livre des Nombres : récit, histoire et loi en Nb. 13, 1-20, 13, (Orbis Biblicus et Orientalis 157), Fribourg-Göttingen, 1997 et surtout, dernièrement, Ulrich Fistill, Israel und das Ostjordanland, (Österreichische Biblische Studien 30), Frankfurt, 2007.

20. Fistill voit dans le second un cas attesté d'intertextualité allant de Dt 3, 1-3 vers $\mathrm{Nb}$ 21,33-35, mais il voit pour le premier des influences diverses dont Jérémie 48, 45-47 : U. Fistill, Israel und das Ostjordanland, p. 60-73.

21. Ce qui n'est pas la position de L. Perlitt, «Deuteronomium 1-3 im Streit der exegestischen Methoden ", Deuteronomium-Studien, p. 109-122, qui pense à un auteur connaissant les traditions parallèles de Nombres et de Josué pour Dt 1-3.

22. U. Fistill, Israel und das Ostjordanland, p. 218 et 220.

23. Littéralement, en hébreu (mōšlîm) et surtout en grec (ainigmatistai), les « déchiffreurs d'énigmes ».

24. Voir, pour cette suggestion, la récente étude d'Étienne Nodet, «Pâque, Azymes et théorie documentaire », Revue biblique, 114, 2007, p. 499-534. 
Quoi qu'il en soit de ces traditions ${ }^{25}$, le vocabulaire utilisé est peut-être seul à même de les situer les unes par rapport aux autres. Or, si $N b$ 13, 33 et $D t$ 1, 28 évoquent tous deux les Anaqim, l'un dans le récit de l'exploration de la région d'Hébron, l'autre à titre de comparaison avec les Amorites, seul le premier les nomme nephilim (gigantes dans la LXX), "hommes de grande taille» (v. 32), le second se contentant de signaler qu'il s'agit d'un « peuple grand et fort ». D'une part, il est à noter que la mention de $N b 13,32$ faisant de ces Anaqim des géants dévorant les habitants du pays se retrouve en 1Hénoch 7, 4-ils se dévorent même entre eux (v. 5) alors qu'elle n'est pas présente dans le Deutéronome ni ailleurs dans les textes bibliques. D'autre part, la série de peuples comparables aux Anaqim cités en Deutéronome - les Émim (Dt 2, 10-11) et les Zamzumim (Dt 2, 20-21) - sont appelés rephâ'îm (LXX : Raphaïn). Il y a donc en Nombres une équivalence entre Anaqim et Nephilim, et une équivalence en Deutéronome entre Anaqim et Rephaïm. Or, si le premier qualificatif est presque un hapax, présent dans deux passages qui ne peuvent être que tardifs, dans des livres eux-mêmes tardifs, le second est, en revanche, très présent dans les textes bibliques et même authentifié ailleurs, notamment dans les tablettes syriennes d'Ougarit (XIII ${ }^{\mathrm{e}}$ siècle av. n.è.. ${ }^{26}$. Autrement dit, les hommes d'antan de haute taille étaient traditionnellement désignés par le terme de rephâ'îm, celui de nephilîm étant sans doute plus conjoncturel. Quant aux Anaqim, terme non générique désignant un «peuple », on ne les trouve mentionnés que de Nombres à Josué. En $D t 9,1-2$, passage appartenant au premier encadrement textuel du code deutéronomique (Dt $12-25)^{27}$ et donc probablement le plus ancien, il est également question des fortifications de leurs villes, celles-ci allant jusqu'au ciel. Surtout, les Anaqim sont seuls nommés parmi les peuples habitant au-delà du Jourdain, terre à conquérir, cela en précisant qu'on ne peut leur résister. Dt 1,28 , qui reprend presque exactement le passage, nuance quelque peu cette

25. Traditions qui se répètent en se faisant écho de Genèse à Josué comme autant de rappels d'un épisode qui devient obsédant, à tel point que la « reconnaissance » de Canaan commence dès les pérégrinations d'Abraham et que la préparation de la conquête prend plus d'ampleur que la conquête elle-même.

26. Voir pour les Rephaïm d'Ougarit et les mentions bibliques, André Caquot, « Rephaïm », Supplément au Dictionnaire de la Bible, X (1985), col. 344-357.

27. Martin Rose, « Deutéronome », T. Römer, J.-D. Macchi, C. Nihan, Introduction à l'Ancien Testament, p. 211-227. 
présence et, pour finir, $N b$ 13, 22 la situe très localement à Hébron, sans parler de fortifications allant jusqu'au ciel, et nomme trois fils d'Anaq : Ahimân, Shéshaï et Talmaï $(N b 13,22)^{28}$.

Ainsi, le motif de mythe de la conquête évolue de diverses manières quand on part de Deutéronome vers Nombres : on va de l'insistance des villes aux fortifications gigantesques à l'insistance concernant les géants eux-mêmes : «Et là nous avons vu les géants, et nous étions en face d'eux comme des sauterelles » $(N b 13,33)^{29}$; de même, on va d'une imprécision géographique et ethnique patente (Dt 9) vers une amplification des récits et l'ajout de détails divers (Dt 1-3/Nb 13; 21; 31). D'ailleurs, si l'on doit évoquer une version ancienne de la conquête, il faut songer au passage d'Amos 2, 9-10 qui parle de la «montée d'Égypte » et qui décrit «l'Amorite» comme un géant: " haut comme des cèdres et fort comme des chênes ${ }^{30}$ ». On le voit, l'évocation est moins spectaculaire et plus poétique. Nous pouvons dès lors penser que l'implication de géants dans le récit de la conquête n'est intervenue que lorsque les auteurs deutéronomiques ont voulu amplifier le mythe. Autrement dit, la piste des géants s'interrompt là, il nous faut désormais suivre celle des Rephaïm.

28. Faut-il voir en ces noms une création de l'auteur de $N b 13$ ou l'évocation d'un lointain passé qu'attesterait le fait que deux de ces noms semblent être hourrites : Edward Lipinski, "'Anaq - Kiryat'Arba' - Hébron et ses sanctuaires tribaux », Vetus Testamentum, 24, 1974, p. 41-55?

29. Le texte massorétique insiste plus encore que la Septante en énonçant à deux reprises le terme nephilîm : « Et là nous avons vu les géants, fils d'Anaq, descendance des géants... ». Cette répétition, ainsi que la précision «fils d'Anaq », nous fait préférer la leçon grecque plus courte.

30. Souvent jugé comme non originel, ce passage (v. 9-12) peut toutefois être considéré comme authentique car il n'emploie pas le verbe « sortir », comme on le trouve dans les passages deutéronomistes, mais « monter » comme en Osée 12, 14 : « et par un prophète, Yhwh a fait monter Israël d'Égypte ». Voir Robert A. Kluger, "The Deuteronomists and the Latter Prophets», L.S. Schearing, S.L. McKenzie (éd.), Those Elusive Deuteronomists. The Phenomenon of Pan-Deuteronomism, (Journal for the Study of the Old Testament Supplement series 268), Sheffield, 1999, p. 127-144 (spécialement p. 136). De même, la mention de l'Amorite au singulier, et non des Amorites ou des Anaqim comme en Deutéronome et en Nombres, indique qu'il s'agit probablement d'une évocation plus ancienne du mythe. Si on l'attribue au voyant Amos, il s'agit alors d'une conception du vIII ${ }^{\mathrm{e}}$ siècle av. n.è., que la mention des quarante ans au désert, en apparence deutéronomiste, ne contraint pas à dater le passage plus tardivement puisque ce topos mythique des quarante ans se trouve déjà dans la stèle moabite de Mésha datant du $\mathrm{IX}^{\mathrm{e}}$ siècle : Niels Peter Lemche, The Israelites in History and Tradition, London-Louisville, 1998, p. 45. 


\section{ÉSAÏE 14 eT ÉZÉCHIEL 32 : REPHA'IM ET GIBboRIM}

Les données bibliques sur les Rephaïm semblent à première vue contradictoires puisque, outre les géants entrevus, on trouve le terme pour désigner des lieux (plaine et vallée des Rephaïm) et pour désigner les habitants du séjour des morts (Shéol). C'est pourquoi il semblait logique de distinguer ces Rephaïm, simples esprits des morts, des géants d'antan, d'autant plus qu'une inscription phénicienne bilingue traduit le terme par " Mânes $^{31}$ ». Les données ougaritiques plus anciennes ont permis d'avancer sur ce sujet et de retrouver le lien existant entre les héros d'autrefois et les esprits des morts; de même que certaines données phéniciennes ${ }^{32}$. Un peu comme en Grèce, les glorieux ancêtres ainsi que les rois d'Ougarit étaient «héroïsés » en entrant dans la catégorie des rapa'ūma et recevaient à ce titre un culte ${ }^{33}$. Or un texte biblique du VIII ${ }^{\mathrm{e}}$ siècle, celui du prophète Ésaïe, donne un contexte et une conception similaires aux tablettes syriennes. Le roi de Babylone étant mort, il rejoint tous les rois qui séjournent dans le Shéol :

«Le séjour des morts s'ébranle pour toi à l'annonce de ta venue.

Pour toi, il réveille les trépassés (rephâ 'îm ${ }^{34}$, tous les grands de la terre, il fait lever de leurs trônes tous les rois des nations... » $(E s \text { 14, 9 })^{35}$.

Ainsi, il apparaît que les Israélito-Judahites, à l'instar de leurs voisins, ne concevaient pas la mort comme la fin de tout mais comme le lieu d'un nouveau séjour dans lequel les rois avaient un statut particulier.

Cette conception se retrouve dans le chapitre 32 du livre du prophète Ézéchiel, moins ancien ${ }^{36}$ mais bien plus suggestif encore que

31. A. Caquot, « Rephaïm », col. 347-348.

32. Il est notamment question d'un lieu de repos avec les Rephaïm : H. Donner, W. Röllig, Kanaanäische und Aramische Inschriften. Band II : Kommentar, Wiesbaden, 1973, p. 17-23.

33. Voir, notamment, Baruch A. Levine, Jean-Michel de Tarragon, « Dead Kings and Rephaim : the patrons of the Ugaritic Dynasty ", Journal of American Oriental Society, 104, 1984, p. 649-659. La nature de ce culte est difficile à déceler mais le sens du verbe râphâ' d'où provient le substantif est presque sans équivoque : " guérir ».

34. La Septante traduit par hoi gigantes.

35. Pour la confrontation de ces données, voir Hedwige Rouillard-Bonraisin, "L'énigme des refa'îm bibliques résolue grâce aux rapa'ūma d'Ougarit », J.-M. Michaud (dir.), La Bible et l'héritage d'Ougarit, Québec, 2005, p. 145-182.

36. Son élaboration va du début $d u$ vI ${ }^{e}$ siècle jusqu'à l'époque perse, peut-être même jusqu'à l'époque hellénistique pour certains passages. Les oracles contre les 
le texte précédent. Après avoir prédit l'anéantissement de l'Égypte et de sa multitude (vv. 1-16), par l'intermédiaire du roi de Babylone, Yhwh annonce au prophète qu'il l'enverra avec les Égyptiens dans le Shéol. Là, il pourra voir tous ceux que le dieu a anéantis avant : les Assyriens, les Élamites, ceux de Mèshèk et de Toubal (Asie Mineure), ceux d'Edom et les gens de Sidon. Il n'y a pas de polémique religieuse dans ce texte. Bien qu'il s'agisse d'étrangers, l'auteur semble partager une même croyance :

«Ils lui [au Pharaon] ${ }^{37}$ parleront, les dieux ('ly) gibbôrîm, du milieu du Shéol avec ses [hommes] forts, ils sont descendus, ils sont couchés les incirconcis, transpercés par l'épée » (v. 21).

Dans les traductions courantes, on rend 'él au pluriel tout autrement : « les plus puissants » (Bible de Jérusalem), «les chefs » (TOB). L'expression a de quoi surprendre mais il s'agit bien de « dieux », comme on trouve ilnym pour qualifier les Rephaïm à Ougarit $^{38}$. Autrement dit, les gibbôrim morts au combat sont devenus les dieux du Shéol. On peut donc les rapprocher des Rephaïm et en déduire que ceux-ci pouvaient être d'anciens Gibborim, c'està-dire des héros, des grands guerriers. Et si la Septante n'a pas dans son texte hoi theoi, en revanche, elle rend gibbôrim par hoi gigantes, comme elle rend souvent rephâ 'îm. La traduction grecque des livres bibliques induit donc une équivalence entre Nephilim, Rephaïm et Gibborim.

Plus intéressant encore, il est dit plus loin :

« Ils [les guerriers de Mèshèk et de Toubal] ne sont pas couchés avec les gibbôrim tombés (nplym) autrefois ${ }^{39}$, qui sont descendus au Shéol avec leur équipement de guerre et à qui on a placé leur épée sous leur tête, leur bouclier ${ }^{40}$ sur leurs ossements car la terreur des gibbôrîm [était] dans la terre des vivants » (v. 27).

nations ( $E z$ 25-32) ont dû être intégrés au début de l'époque perse mais avec des prolongements plus tardifs. Voir C. Nihan, « Ezéchiel», T. Römer, J.-D. Macchi, C. Nihan, Introduction à l'Ancien Testament, p. 359-378 (spécialement p. 371-372).

37. La Septante a : «Ils te parleront. »

38. Voir le "poème des Rephaïm» dans André Caquot, Maurice Sznycer, Andrée Herdner, Textes Ougaritiques I. Mythes et légendes, (Littératures anciennes du Proche-Orient 7), Paris, 1974, p. 459-480.

39. Septante : ap aiônos = mé ôlām au lieu de mé ărélîm «les incirconcis » (texte massorétique).

40. Il est plus logique de lire ainsi (comme le fait la Bible de Jérusalem) : \nwtm au lieu de 'nwtm, « leur faute » (texte massorétique), les lettres tsadé et 'ayin ayant une graphie proche portant à confusion. 
Ce passage distingue les héros d'antan, qui avaient plus de valeur que les guerriers des puissances d'alors, et nous ramène aux Nephilim de Genèse 6 par l'emploi du participe du verbe nâphal : les «tombés » (nōphlîm) ${ }^{41}$. On aurait donc ici «le chaînon manquant $»$ : l'emploi de nephilîm s'expliquerait par le fait que les géants d'antan étaient les héros « tombés » dans le Shéol. Et nous aurions une notion commune dans ces textes prophétiques plus anciens et dans les textes tardifs du Pentateuque: les gibbôrîm deviennent des rephâ'îm une fois tombés sur le champ de bataille, le terme de nephilim condensant en quelque sorte les deux autres. C'est l'entrée dans le Shéol qui conditionne l'appartenance aux Rephaïm; quant à l'aspect gigantesque des héros, il semble que ce soit lié à leur caractère légendaire.

\section{SAMUel 21 et 23 : Gibborim et SHALISHim}

Dans l'ensemble $D t$ 2-3, il y a une interpolation explicative concernant $\mathrm{Og}$, roi du Bashân - région transjordanienne bordant le lac de Tibériade et correspondant au Golan actuel -, qui donne à penser qu'il était un personnage légendaire connu dans toute la région $^{42}$, un peu comme le prophète Balaam ( $\left.\mathrm{Nb} 22-24\right)$ dont l'existence a été confirmée historiquement par l'inscription « ammonite » de Deïr 'Alla (vIII' siècle) ${ }^{43}$. En effet, en plus d'être mentionné dans une inscription funéraire de Byblos ${ }^{44}, \mathrm{Og}$ est dit : «dernier des

41. On ne peut vocaliser autrement et lire nephilim comme le propose Jan Alberto Soggin, « Sons of God(s), Heroes and nephilim : Remarks on Genesis 6 : 1-4 », M.V. Fox, V. Avigdor Hurowitz, A. Hurvitz, M.L. Klein, B.J. Schwartz, N. Shupak (éd.), Texts, Temples and Traditions. A Tribute to Menahem Haran, Winona Lake, 1996, p. 135-136, car la Septante a traduit par le participe parfait du verbe piptô, « tomber ».

42. Et même au-delà? voir Scott. B. Noegel, « The Aegean Ogygos of Beotia and the Biblical Og of Bashan : Reflections of the Same Myth », Zeitschrift für die Alttestamentliche Wissenschaft, 110, 1998, p. 411-426.

43. Voir la notice de B.A. Levine dans W. Hallo, K. Lawson Younger (éd.), The Context of Scripture II. Monumental Inscriptions from the Biblical World, Leiden-Boston-Köln, 2000, p. 140-145.

44. Fragment d'un sarcophage du ver-IV siècle, l'inscription évoque «le $\mathrm{Og}$ » qui viendra chercher le défunt pour l'emmener, probablement, dans le lieu de repos des Rephaïm. Voir Jean Starcky, « Une inscription phénicienne de Byblos », Mélanges de l'Université Saint-Joseph, 45, 1969, p. 259-273. 
Rephaïm ${ }^{45}[\ldots]$, son lit en fer, à Rabba des Ammonites, était long de neuf coudées et large de quatre » $(D t 3,11)$, soit environ 4 mètres 50 sur 2 mètres $^{46}$. Ces dimensions gigantesques ne sont pas sans nous faire penser à un personnage bien plus connu dans la Bible : Goliath. Or celui-ci est d'une taille moins impressionnante : quatre coudées et un empan, soit simplement plus de deux mètres, et il n'est rien dit sur sa largeur. On le voit, la tendance a été à l'exagération des dimensions de ces personnages, d'autant plus que la version massorétique de 1Samuel 17, 4 mentionne six coudées, cela contre les autres témoins plus anciens (Septante, Flavius Josèphe et un manuscrit de Qoumrân) ${ }^{47}$.

Le récit concernant le combat entre David et Goliath ne peut être considéré comme étant originel. En effet, il existe une notice plus brève qui ne fait plus état de la taille du Philistin mais seulement de sa lance aussi lourde qu'une ensouple de tisserand (2Samuel 21, $19=1$ Samuel 17, 7). Le récit plus bref et la description presque inexistante du géant font de ce texte l'origine de l'exploit de David ${ }^{48}$, d'autant plus que si le héros israélite est toujours de Bethléem $(2 S 21,19=1 S 17,12)^{49}$, l'auteur de l'exploit n'est plus David mais un certain Elhanan. La fin du deuxième livre de Samuel est d'ailleurs particulièrement intéressante car le récit général se conclut avec divers textes qui ont peu de lien entre eux. Certains semblent avoir été composés pour clore le rouleau, comme le «psaume » de David (2S 22), d'autres semblent des fragments anciens insérés. C'est donc le cas de $2 S 21,15-22$, qui mentionne simplement les " serviteurs » ('ăbâdîm) de David qui durent combattre ses ennemis afin de protéger leur roi contre les héros des Philistins. Le passage semble donc

45. De même en Josué 12,4 et 13, 12.

46. Voir Ulrich Hübner, « Og von Baschan und sein Bett in Rabbat-Ammon (Deuteronomium 3, 11) », Zeitschrift für die Alttestamentliche Wissenschaft, 105, 1993, p. 86-92.

47. Bernard Grillet, Michel Lestienne, Premier livre des Règnes, (La Bible d'Alexandrie 9.1), Paris, 1997, p. 295.

48. Contrairement à A. Graeme Auld, «The Story of David and Goliath : A Test Case for Synchrony plus Diachrony », W. Dietrich (Hg.), David und Saul im Widerstreit - Diachronie und Synchronie im Wettstreit : Beiträge zur Auslegung des ersten Samuelbuches, (Orbis Biblicus et Orientalis 206), Göttingen, 2004, p. $118-128$.

49. Le personnage de David est d'ailleurs chargé idéologiquement dans ce récit car il est à la fois de Bethléem en Juda et d'Éphraïm, c'est-à-dire à la fois du Sud et du Nord, futur roi idéal d'un royaume d'Israël unifié. 
avoir été réécrit car l'explication selon laquelle David ne combattit pas ses ennemis directement parce qu'il était fatigué ${ }^{50}$ (v. 15), ou pour que ne s'éteigne pas « la lampe d'Israël» (v. 17), n'est pas très convaincante. En outre, il est probable que Yishbi-be-Nob, Saf, Goliath et l'inconnu ayant six doigts à chaque main et six orteils à chaque pied n'aient pas été des Philistins à l'origine puisqu'ils sont dits « descendants de Râphâh » (vv. 16. 18. 20. 22), la Septante parlant même des « enfants des géants de Rapha ». Malgré la différence de graphie de la troisième lettre, il est assez évident qu'il s'agit là non de Philistins mais bien de Rephaïm possédant des armes de géants, d'autant plus que le passage parallèle de 1Chroniques 20, 4-8 emploie ce terme ${ }^{51}$, et que les héros qui les abattirent n'avaient pas forcément de lien avec David au départ.

L'autre série de faits guerriers à la fin de ce livre ne semble pas avoir subi un même travail rédactionnel tant le texte de $2 S 23$, 8-39 est en mauvais état ${ }^{52}$. Seuls les versets 13 à 17 semblent être rédactionnels puisqu'ils font intervenir David dans un rôle tout aussi passif et inutile qu'en $2 S 21$. Cette fois, les héros israélites reçoivent le terme de gibbôrîm mais, là encore, il s'agit vraisemblablement d'une désignation extérieure au texte lui-même, donc plus tardive. En fait, le texte parle de šlš et de šlšym que la Septante traduit tout naturellement par « trois » (treis) et par « trente » (triakonta). Mais sans doute faut-il rapprocher ce terme de šlyš, traduit en grec par tristatês et désignant un soldat spécifique ${ }^{53}$. Il est évident que le terme a quelque chose à voir avec, d'une part le chiffre trois, d'autre part la charrerie du fait des contextes dans lequel on le trouve ${ }^{54}$. Cependant, les « héros de David» ne sont

50. La Septante a «il s'en alla » (!).

51. André Caquot, Philippe de Robert, Les Livres de Samuel, (Commentaire de l'Ancien Testament 6), Genève, 1994, p. 587.

52. Ibid., p. 620.

53. Ibid., p. 621 et 625. Faut-il comprendre qu'il s'agissait d'un " chevalier» sur un char, en se basant sur d'autres données sémitiques - Marc Vervenne, « Hebrew ša $\bar{l} l \hat{s}$ - Ugaritic t $\underline{\text { tlt }}$ », Ugarit-Forschungen, 19, 1987, p. 355-373 -, ou d'un « officier de troisième rang »-B.A. Mastin, "Was the šălišs the Third Man in the Chariot? », J.A. Emerton (éd.), Studies in the Historical Books of the Old Testament, (Supplements to Vetus Testamentum 30) Leiden, 1979, p. 125-154. À moins qu'il ne s'agisse de guerriers combattant par trois : Donald G. Schley, « The šălî̌sim, Officers of Special Three-Men Squads? », Vetus Testamentum, 40, 1990, p. 321-326.

54. Voir Exode 14, 7 et 15, 4; 2Rois 7, 2. 17-19; 9, 25; 10, 25. 
pas sur des chars, on pourrait donc en déduire que le terme leur fut appliqué a posteriori. À moins de penser que le šâlîs ne soit devenu un soldat de la charrerie à cause de l'adoption de cet équipement par le roi, mais tout en restant un soldat d'élite au service de celui-ci (2Rois 9, 24-25). L'expression qui semble la moins contestable du texte est $r$ 'š hšlšy et est attribuée à deux héros : Ishbaal ${ }^{55}$ et Abishaï, " élite » des Shalishim ( $2 S 23,8$ et 18). Ainsi, en parlant des « trois grands guerriers » (̌̌elōšèt haggibōrîm) et en transformant le terme militaire šâlî̌sîm en simple šelōšìm, " trente », le rédacteur introduisant le texte a cherché à lui donner une cohérence qu'il n'avait probablement pas à l'origine ${ }^{56}$. Il n'a sans doute pas fait que cela car la présence d'Abishaï et de Benayahou est douteuse, le premier intervenant beaucoup aux côtés de David dans des récits moins anciens, le second apparaissant dans les récits d'accession au trône de Salomon ${ }^{57}$. Mais, quoi qu'il en soit de l'identité des héros et du sens originel du terme Shalish ${ }^{58}$, les exploits conservés sont saisissants : Ishbaal fait neuf cents victimes en une seule fois $(\mathrm{v} .8)^{59}$, « Abishaï » six cents (v. 18) ${ }^{60}$ et « Benayahou », quant à lui, frappe deux héros ${ }^{61}$ de Moab, tue un lion dans une citerne et abat un Égyptien probablement de grande taille ${ }^{62}$ et à armes inégales (vv. 20-21). Contrairement à ce que l'on peut encore affirmer ${ }^{63}$, les exploits de ce type ne peuvent être attribués à de simples soldats du roi David, ils sont bien plus les hauts faits de grands guerriers légendaires. D'ailleurs, les deux textes pouvaient ne pas être distincts à

55. Restitution à partir de la LXX. Voir Dominique Barthélemy, Critique textuelle de l'Ancien Testament, (Orbis Biblicus et Orientalis 51/1), GöttingenFribourg, 1982, p. 311.

56. Cohérence plus apparente dans le passage parallèle de 1Chroniques 11, 10-41, notamment par l'établissement de trente noms dans la liste finale, et non de trente-sept comme en $2 S 23$.

57. D. Barthélemy, Critique textuelle de l'Ancien Testament, p. 319-320.

58. Il semble que šlš résiste même à une recherche étymoniale biconsonantique : Georges Bohas, Mihai Dat, Une théorie de l'organisation du lexique des langues sémitiques : matrices et étymons, Lyon, 2007, p. 79.

59. D'après la Septante (texte antiochien) et Flavius Josèphe (Antiquités Juives VII, 308); le texte massorétique indique « seulement » huit cents.

60. LXX ${ }^{L}$ et Josèphe (Antiquités Juives VII, 315) contre le TM : trois cents.

61. Il y a une difficulté à interpréter le terme 'ări'él, que la Septante a «traduit» par « fils d'Ariel».

62. Littéralement : ayant « belle allure» (mare'èh/horatos).

63. Voir, par exemple, l'étude de Herbert H. Klement, II Samuel 21-24. Context, Structure and Meaning in the Samuel Conclusion, (Europäische Hochschulschriften XXIII/682), Frankfurt, 2000. 
l'origine car Elhanan de Bethléem en $2 S$ 21, 19, qui battit Goliath de Gat, se retrouve dans la liste des « trente » en $2 S 23,24$, comme étant Elhanan fils de Dodo de Bethléem. Sans doute doit-on alors le restituer au verset 9 à la place d'Eléazar, auteur d'un exploit contre les Philistins et fils de Dodo lui aussi. Autrement dit, il pouvait y avoir un texte-source qui fut diversement utilisé ou, pour le moins, des traditions communes.

\section{Shamma, Shamgar et Samson}

Parmi les Gibborim de David, il en est un qui ne semble pas avoir subi de remaniements puisqu'il est fait mention de son exploit et qu'il occupe la troisième place dans la liste des « trente » avec plus ou moins la même ascendance $(2 S 23,25)$. Il s'agit de Shamma le Hararite qui défit les Philistins, seul au milieu d'un champ cultivé, et cela à Lehi (v. 11). Ce type d'exploit se retrouve en Juges 3, 31 où un dénommé Shamgar Ben Anath battit six cents Philistins avec « un aiguillon à bœufs ». Or on retrouve ce personnage mentionné dans ce que l'on peut appeler le plus ancien texte biblique : le chant de Deborah (Juges 5, 6) ${ }^{64}$. Mais, surtout, l'exploit fait irrémédiablement penser à celui de Samson, là encore contre les Philistins et à Lehi même (Juges 15, 14-15) :

«Lui vint à Lehi et les Philistins exultaient en allant vers lui. Alors le souffle de Yhwh fondit sur lui [...] et il trouva une mâchoire d'âne jetée sur le chemin ${ }^{65}$ et il étendit sa main, la prit et frappa avec elle mille hommes. »

La parenté entre les deux derniers héros est d'autant plus troublante que l'on retrouve le nom du dieu solaire dans Šamšôn - Shamash -, sous sa forme sémitique ${ }^{66}$, et dans $\check{S}$ amgar - Šimigi -

64. Les archaïsmes de langage le feraient remonter au $x^{e}$ siècle av. n.è. Voir Ernst Axel Knauf, «Deborah's Language Judges Ch. 5 in its Hebrew and Semitic Context », B. Burtea, J. Tropper, H. Younansardaroud (Hg.), Studia Semitica et Semitohamitica, (Alter Orient und Altes Testament 317), Münster, 2005, p. 167-182.

65. Cette leçon de la Septante est préférable à celle du texte massorétique qui fait mention d'une mâchoire " fraîche » voulant induire que Samson commettait un acte d'impureté en saisissant un morceau de cadavre.

66. Nous préférons la vocalisation de la Septante (Sampsôn) à celle du texte massorétique (Šimšôn) car elle est comparable à celle de Salomon : Šlōmōh en 
sous sa forme hourrite ${ }^{67}$. Or, que Samson fût un géant dans un récit plus archaïque que dans sa forme dernière en Juges 13-16 ne fait pas de doute. En effet, l'ensemble de ses exploits et, plus particulièrement, celui qui le fait transporter les portes de la ville de Gaza jusque sur la montagne en face d'Hébron $(16,3)$ révèle une telle figure. Même si le héros fut rajeuni pour illustrer un rite ayant trait aux jeunes hommes ${ }^{68}$ et fut « rapetissé » pour en faire un juge dans une optique de reconstruction historique d' « Israël » $(15,20$ et 16, 31), le personnage garde des allures de géant inspirant la crainte $(14,11)^{69}$.

L'autre aspect intéressant, concernant ces héros qui réalisent des exploits de géants, a trait à la symbolique des chiffres. Nous avons relevé les nombres de victimes impressionnants contenus en 2Samuel 23 et avons choisi les leçons du grec contre l'hébreu car elles font apparaitre une symbolique qui trouve des appuis ailleurs. Ainsi, Ishbaal vainc 900 hommes et Abishaï 600, soit des multiples de 3. Shamgar en abat lui aussi 600 et, quant à Samson, si l'on fait fi des relectures du récit qui ont naturellement introduit la symbolique israélite bien connue du chiffre $7^{70}$, on se rend compte que ses exploits vont grandissant : d'abord il tue 30 hommes d'Ashqelon (Jug 14, 19), puis capture 300 renards pour détruire les cultures des gens de Timna $(15,4)$, à Lehi, il défait 1000 Philistins avec la simple mâchoire d'âne $(15,15-16)^{71}$, et, pour finir, prisonnier et

hébreu, mais Salômôn en grec; le fils de David aurait donc un nom théophore similaire, avec le suffixe - ôn, se référant au dieu de Jérusalem : Shalem.

67. Emmanuel Laroche, «Glossaire de la langue hourrite. Deuxième partie », Revue hittite et asianique 35, 1977, p. 232-233.

68. Voir notre étude, «Samson le nazir : un mythe du jeune guerrier», Revue de l'histoire des religions 222, 2005, p. 259-286.

69. En suivant le texte de la Septante et la paraphrase de Flavius Josèphe (Antiquités juives $\mathrm{V}, 289$ ); le texte massorétique ayant simplement : «comme ils le virent ».

70. Notamment dans le récit de l'énigme (Jug 14, 15-18) et, plus encore, dans le récit à l'allure de conte littéraire mettant en scène Samson et la fameuse Dalila et qui occupe pas moins de quinze versets. D'abord, il lui indique de le lier avec 7 cordes, puis elle fait à sa demande 7 tresses.

71. Au point de pouvoir clamer avec une ironie macabre qu'il a fait deux tas de morts et, ainsi, de nommer le lieu «Ramat Lehi », c'est-à-dire " hauteur de la mâchoire ». En effet, le vocable hmmr a un double sens selon sa vocalisation :

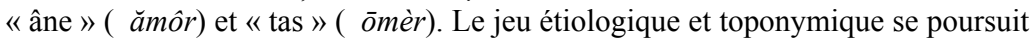
même puisque, mourant de soif et Elohim (?) faisant surgir une source du rocher, Samson la nomme "Source de la mâchoire » $(\mathrm{LXX})$ - et non « Source du suppliant » comme le propose l'hébreu (TM) pour harmoniser le texte. 
aveugle à Gaza, tel un géant il enterre 3000 hommes et femmes sous les ruines du temple de Dagon $(16,27-30)$. La présence du chiffre 3 est telle dans le texte que l'on peut même tenter d'isoler trois fautes majeures et distinctes (« fonctionnelles ») commises par Samson, comme Georges Dumézil l'a fait pour Héraklès ${ }^{72}$. Autrement dit, les héros légendaires ouest-sémitiques conservés dans les textes et les fragments anciens de la Bible, ou ayant une origine ancienne, illustrent une fonction guerrière très présente dans la culture israélito-judahite ${ }^{73}$ et qui était symbolisée par le chiffre 3 et ses multiples, ce qui nous ramène de nouveau au šâlî̌s sans pour autant savoir ce que ce terme recouvrait exactement.

\section{Conclusion}

La fonction guerrière dans le monde israélito-judahite, difficile à reconstituer tant les textes ont pu être modifiés et tant l'histoire construite d'Israël est prégnante - d'Adam à Josué en passant par les «pacifiques » Abraham et Moïse -, est donc complète puisqu'elle fait intervenir les jeunes guerriers rusés, ba®ûrîm et nezirîm ${ }^{74}$, et les guerriers aguerris, gibbôrîm et šâlišsîm. À leur mort, ces derniers devenaient des personnages légendaires dont on grandissait les exploits ainsi que leur taille, ils étaient mêmes des dieux dans le royaume des morts en étant appelés rephâ 'îm, terme qui évoluera à la fin de l'époque perse en nephilîm dans un sens moins précis et moins religieux. Autant dire tout de suite que les géants que l'on trouve en 1Hénoch, dans Jubilés ou encore dans le Livre des Géants, dont les fragments ont été retrouvés dans les grottes de Qoumrân,

72. Voir l'étude récente de Bernhard Lang, «The Three Sins of Samson the Warrior », I. Kottsieper, R. Schmitt, J. Wöhrle (Hg.), Berührungspunkte. Studien zur Sozial- und Religionsgeschichte Israels und seiner Umwelt, (Alter Orient und Altes Testament 350), Münster, 2008, p. 179-192.

73. La plupart des toponymes mentionnés dans la liste finale de $2 S 23$ sont judéens : A. Caquot, P. de Robert, Les Livres de Samuel, p. 624.

74. Voir nos positions de thèse sur cette question : «Etre nazir : du guerrier yahwiste au vœu cultuel du judaïsme ancien. Origine et transformation d'un rite de cheveux », Revue de l'histoire des religions, 224, 2007, p. 275-288. La catégorie « nazir » est très spécifique et pouvait traverser les autres puisque Samson comme Saül - C. Lemardelé, "Saül le nazir ou la légende d'un roi », Scandinavian Journal of the Old Testament, 22, 2008, p. 47-62 - étaient saisis par l'esprit de Yhwh pour des exploits de jeune guerrier comme pour des exploits de grand guerrier. 
n'ont plus grand-chose à voir avec la fonction guerrière. Ils sont bien plutôt le développement théologique du motif de Genèse 6, 1-4 qui, nous l'avons vu, ne fait intervenir des géants que de manière allusive. Les auteurs s'inspirant de ce passage ont donc considérablement détourné et amplifié le motif. D'une part, les géants sont désormais clairement issus de l'union illicite entre les fils de Dieu et les filles d'homme, d'autre part, ils sont aussi « hauts de trois mille coudées » (!) et cannibales (1Hénoch 7, 1-6).

L'arrière-plan héroïque et mythique que nous avons mis en évidence est, de même, très différent du mythe grec de la gigantomachie dans lequel les dieux et les géants se livrent un combat déterminant pour l'humanité et la civilisation ${ }^{75}$. Quant au monde mésopotamien, il ne nous semble pas qu'il faille puiser dans le corpus mythique à l'exception de l'Épopée de Gilgamesh, héros de taille gigantesque et roi d'Uruk à l'origine ${ }^{76}$. En effet, les récits du déluge dans leurs différentes versions n'évoquent pas tant une faute humaine qu'un désir des dieux d'en finir avec l'homme ${ }^{77}$; tout comme d'ailleurs dans la Genèse avant l'insertion des fils de Dieu et des filles d'homme.

7 Rond-Point des Sans-Soucis

78340 Les-Clayes-sous-Bois

ch.lemardele@laposte.net

75. Pour ce mythe essentiellement connu grâce à l'iconographie, voir l'ouvrage déjà ancien de Francis Vian, La Guerre des géants : le mythe avant l'époque hellénistique, Paris, 1952.

76. Raymond-Jacques Tournay, Aaron Schaffer, L'Épopée de Gilgamesh, (Littératures Anciennes du Proche-Orient 15), Paris, 1994.

77. Jean Bottéro, Samuel Noah Kramer, Lorsque les dieux faisaient l'homme. Mythologie mésopotamienne, Paris, 1989, p. 526-601. 